Research Article

\title{
Effects and Mechanism of Zishen Jiangtang Pill on Diabetic Osteoporosis Rats Based on Proteomic Analysis
}

\author{
Shufang Chu, ${ }_{1}^{1}$ Deliang Liu, ${ }^{1}$ Hengxia Zhao, ${ }^{1}$ Mumin Shao, ${ }^{2}$ Xuemei Liu, ${ }^{1}$ Xin Qu, \\ Zengying Li, ${ }^{1}$ Jinhua Li, ${ }^{1}$ and Huilin $\mathrm{Li} \mathbb{1}^{1}$ \\ ${ }^{1}$ Department of Endocrinology, Shenzhen TCM Hospital, \\ The Fourth Clinical Medical College of Guangzhou University of Chinese Medicine, Shenzhen 518033, China \\ ${ }^{2}$ Department of Pathology, Shenzhen TCM Hospital, \\ The Fourth Clinical Medical College of Guangzhou University of Chinese Medicine, Shenzhen 518033, China \\ Correspondence should be addressed to Huilin Li; sztcmlhl@163.com
}

Received 30 April 2021; Revised 5 July 2021; Accepted 6 August 2021; Published 25 September 2021

Academic Editor: Li Zhang

Copyright (C) 2021 Shufang Chu et al. This is an open access article distributed under the Creative Commons Attribution License, which permits unrestricted use, distribution, and reproduction in any medium, provided the original work is properly cited.

Context. Zishen Jiangtang Pill (ZJP) is a Chinese herbal compound, which has a positive therapeutic effect on diabetic osteoporosis (DOP) by regulating glucose metabolism and bone metabolism. However, its regulatory role and mechanism are still unclear. Objective. To explore the effect and mechanism of ZJP on DOP rats by proteomic analysis. Materials and Methods. After the establishment of diabetes model by Streptozocin (STZ, $60 \mathrm{mg} / \mathrm{kg}), 40$ Wistar rats were equally divided into normal group, model group (diabetic rats), high-dose group $(3.0 \mathrm{~g} / \mathrm{kg} / \mathrm{d}$ ZJP), and low-dose group $(1.5 \mathrm{~g} / \mathrm{kg} / \mathrm{d} \mathrm{ZJP})$ and received treatment for $3 \mathrm{months}$. Histological changes in bone and pancreas tissues were observed by hematoxylin and eosin staining, electron microscopy, and immunofluorescence. Proteomic and bioinformatic analyses were performed to identify the differentially expressed proteins. The fingerprint and active ingredients of ZJP were identified via high-performance liquid chromatography (HPLC). Results. Compared with the model group, ZJP could rescue the weight, fasting blood glucose, and fasting insulin of rats in both high-dose and low-dose group. ZJP could also improve the microstructures of pancreatic islet cells, bone mass, and trabecular and marrow cavities in DOP rats. Bioinformatic analysis suggested that ZJP might influence DOP via multiple pathways, mainly including ribosomes, vitamin digestion and absorption, and fat digestion and absorption. The primary active ingredients, including notoginsenoside R1, ginsenoside Rg1, ginsenoside Re, icariin, and ginsenoside Rb1, were detected. Conclusion. ZJP could significantly improve the histomorphology and ultrastructure of bone and islets tissues and might serve as an effective alternative medicine for the treatment of DOP.

\section{Background}

DOP is a common diabetic complication $[1,2]$. The main pathological changes associated with DOP are a decrease in bone mass per unit volume, a reduction in bone strength, and an increase in bone fragility $[3,4]$. With the aging of the global population, the incidence of DM is increasing every year, which directly leads to an increase in the incidence of DOP $[5,6]$. At present, the treatment of DOP is mainly based on antiosteoporosis drugs $[7,8]$. Western medicine treatments generally include taking calcium, vitamin $\mathrm{D}$, sex hormones, bisphosphonates, calcitonin, and/or fluoride or using monoclonal antibodies [9-11]. However, the therapeutic effects of such drugs are still controversial, and some patients cannot tolerate their side effects or afford their expensive prices. As such, there is an urgent need to identify the mechanism of osteoporosis in patients with diabetes in an effort to identify more cost-effective treatments.

Recently, a large number of experimental and clinical studies detailing the treatment of DOP have affirmed the efficacy of Traditional Chinese Medicine (TCM) [12-14]. Zishen Jiangtang Pill (ZJP) is a TCM compound developed by Shenzhen TCM Hospital. The components of ZJP have the functions of nourishing the breath feminine, nourishing 
the kidneys, and strengthening the bones. Its use is in line with the theory of "kidney dominating bone" for the treatment of osteoporosis. A previous study has found that ZJP has a positive function with regard to hypoglycemic action, decreasing lipids, and improving insulin sensitivity and revealed that ZJP could play an antiosteoporosis role on multiple targets, as based on in vitro experiments [15]. However, the molecular mechanism by which ZJP works is still not fully understood.

Based on a previous study, we have known that ZJP has glycemic control effects with regulating effects on bone metabolism and hypothesized that ZJP can effectively treat DOP rats. In order to explore the effect and mechanism of ZJP on diabetic osteoporosis rats, we established a DOP rat model and treated these rats with different doses of ZJP and observed the bones and histomorphology of pancreatic tissue. By using gene ontology (GO) and Kyoto Encyclopedia of Genes and Genomes (KEGG) analysis, these results reveal that ZJP may affect DOP via multiple pathways.

\section{Materials and Methods}

2.1. Drugs, Reagents, and Antibodies. Zishen Jiangtang Pills (ZJP) were prepared by the Pharmacy Department of Shenzhen TCM Hospital. The main components were Astragali Radix, Rehmanniae Radix Praeparata, Epimedii Folium, Notoginseng Radix et Rhizoma, Codonopsis Radix, Achyranthis Bidentatae Radix, Schisandrae Chinensis Fructus, Polygonati Rhizoma, and Drynariae Rhizoma (Batch No. Guangdong Z20070085). The specific formula composition and preparation method are referred from chen et al [16].

The main reagents and antibodies used are as follows: STZ and carboxymethyl cellulose sodium (CMC) (No. S0130/C4888, Sigma, USA), insulin primary antibody (No. 3014s, CST, USA), fluorescent secondary antibody (No. 111585-003, Jackson ImmunoResearch Laboratories, Inc., USA; and No. A23210, Abbkine, USA), glucagon primary antibody (No. ab10988, Abcam, UK), DAPI fluorescent seal tablets (No. 0100-20, Southern Biotech), chromatographically pure acetonitrile (Merck, USA), and the reference products including notoginsenoside R1, ginsenoside Rg1, ginsenoside Re, icariin, and ginsenoside Rb1 (China Food and Drug Testing Institute). ZJP is evenly mixed and suspended in CMC.

2.2. Animals. A total of 70 2-month-old Specific PathogenFree (SPF) male Wistar rats, weighing from 160 to $190 \mathrm{~g}$, were purchased from the experimental animal center of Southern Medical University with a quality certificate No. 44002100006561. The animals were housed in the SPF room in Shenzhen Municipal Center for Disease Control and Prevention, with an ambient temperature of $18-22{ }^{\circ} \mathrm{C}$, natural circadian rhythm illumination (12 h: $12 \mathrm{~h}$ ), and environmental humidity of $40-70 \%$. The rats were kept in microisolator cages and were given free access to food and water. All animal experiments were in accordance with the Animal Research Ethics Committee and approved by the Animal Protection and Use Committee of the Guangdong
Experimental Animal Center (approval number: IACUCG16003).

2.3. Plasma Measurements. Blood samples were collected from the animals' tail veins. The fasting blood glucose (FBG) levels and fasting plasma insulin (FINS) were determined by test assay kits abiding by the manufacturer's instructions (Roche Diagnostics Co., Ltd., Germany, and USBiological Co., Ltd., United States).

2.4. Bone Mineral Density (BMD) Detection. The BMD of lumbar vertebrae (L1-L4) was detected by using dual-energy X-ray absorptiometry (DEXA) scanning system (GE, United States) on the last day of the experiment. The measurement parameters are dual-energy voltage $41 \mathrm{kVp}$ and $100 \mathrm{kVp}$, scanning window width $18 \mathrm{am}$, and scanning rate $4.8 \mathrm{~s} / \mathrm{am}$.

2.5. Establishment of a Rat Diabetes Model. Rats were adaptively fed for 2 weeks, and 10 rats were randomly selected as normal controls. The remaining 60 rats were used to establish the diabetes model. After fasting for $12 \mathrm{~h}$, rats were injected intraperitoneally with $0.2 \%$ STZ (diluent $0.1 \mathrm{M}$ citrate-sodium buffer, $\mathrm{pH} 4.5$ ) at a dose of $60 \mathrm{mg} / \mathrm{kg}$ [17]. The blood glucose of the rats was measured using a blood glucose meter $72 \mathrm{~h}$ after administration. Rats with a fasting blood glucose $>16.7 \mathrm{mmol} / \mathrm{L}$ were fed commonly for 2 weeks. Those rats that still had a fasting blood glucose $>16.7 \mathrm{mmol} /$ $\mathrm{L}$ were considered as successful models [18]. The normal control group was injected intraperitoneally with the same amount of $0.1 \mathrm{M}$ citrate-sodium buffer $(\mathrm{pH}=4.5)$. In the diabetes induction, 8 animals were dead and 12 animals were failed. The failed animal studies were performed under isoflurane anesthesia and every effort was made to minimize suffering. Finally, a total of four groups with 10 rats in each group were obtained. The rats of four groups then received treatment for 3 months. The normal group and model group were given $0.5 \%$ sodium carboxymethyl cellulose $10 \mathrm{~mL} / \mathrm{kg} /$ day via intragastric administration, and the high-dose $(3.0 \mathrm{~g} /$ $\mathrm{kg} / \mathrm{d}$ ZJP) and low-dose $(1.5 \mathrm{~g} / \mathrm{kg} / \mathrm{d}$ ZJP) groups were simultaneously given $0.5 \%$ sodium carboxymethyl cellulose $10 \mathrm{~mL} / \mathrm{kg} /$ day via intragastric administration. At the end of the treatment, all of the rats were fasted for $12 \mathrm{~h}$ and then anesthetized using sodium pentobarbital $(50 \mathrm{mg} / \mathrm{kg})$ and sacrificed via abdominal aorta bleeding. The blood was obtained, and the pancreas and bone tissues were stored at $-80^{\circ} \mathrm{C}$. After muscle and tendons were removed, the femoral bone was utilized for biochemical and histologic evaluation.

2.6. Hematoxylin and Eosin (H\&E) Staining. Pancreatic tissues were sliced, fixed in $4 \%$ paraformaldehyde, rinsed with water for $24 \mathrm{~h}$, dehydrated with an ethanol gradient, placed in anhydrous acetone, and embedded in polymethyl methacrylate [19]. H\&E staining was performed as follows: xylene for $5 \mathrm{~min}$, xylene for $3 \mathrm{~min}, 100 \%$ alcohol for $30 \mathrm{~s}$, $100 \%$ alcohol for 30 s, $95 \%$ alcohol for 30 s, $90 \%$ alcohol for $30 \mathrm{~s}$, hematoxylin staining for 10-15 min, 1\% hydrochloric acid alcohol differentiated slice for $10 \mathrm{~s}, 1 \%$ eosin staining for 
3 min, $90 \%$ alcohol for 30 s, $95 \%$ alcohol for 30 s (2x), 100\% alcohol for $30 \mathrm{~s}(3 \mathrm{x})$, carbonic acid xylene for $30 \mathrm{~s}$, xylene for 30 s $(3 x)$, and a neutral gum seal.

2.7. Double-Label Pancreatic Immunofluorescence. The paraffin slices were baked at $60^{\circ} \mathrm{C}$, dewaxed with xylene and ethanol, and repaired using citrate antigen repair solution. The slices were then incubated with insulin $(1: 400)$ and glucagon $(1: 1000)$ primary antibodies in the dark at $4^{\circ} \mathrm{C}$ overnight. The slices were then incubated with a fluorescent secondary antibody $(1: 100)$ at $37^{\circ} \mathrm{C}$ in the dark for $1 \mathrm{~h}$, stained with DAPI, and sealed using a fluorescent sealing tablet. The positive islet beta cells appeared red, the positive islet alpha cells appeared green, and the nuclei appeared blue.

\subsection{Pancreatic Transmission Electron Microscopy.} Pancreatic tissues were fixed with $2.5 \%$ glutaraldehyde, dehydrated in ethanol, penetrated with propylene oxide, and embedded with a $1: 1$ propylene oxide and resin mixture for $2 \mathrm{~h}$, pure resin for $2 \mathrm{~h}$, and resin at $48^{\circ} \mathrm{C}$ for $10 \mathrm{~h}$. The samples were then sliced at 500-1000 nm, stained with toluidine blue, sliced at $70 \mathrm{~nm}$, and stained with lead and uranium.

2.9. Femoral Electron Microscope Scanning. The fixed bone tissue sections were processed as follows: sections were stained with Weigert's iron lignin on a glass slide for $40 \mathrm{~min}$ and rinsed with water until it turned blue. These were then incubated in $1 \%$ hydrochloric acid ethanol and washed with water until it turned blue, dyed with Van Gieson picric acidmagenta solution for $3 \mathrm{~min}$, dehydrated with 95\% ethanol and anhydrous ethanol, quickly dipped in fresh anhydrous ethanol, and then blotted dry. After the above treatment, the sections were immersed in an acetonitrile solution, which was then replaced with $70 \%, 80 \%, 90 \%, 95 \%$, and $100 \%$ acetonitrile, soaked for $15-20 \mathrm{~min}$ each time, and finally replaced with $100 \%$ acetonitrile and dried. The sections were then sprayed with carbon and gold and observed under an electron microscope.

2.10. Proteomic Analysis. The proteins were extracted from the rats' bone tissues in different groups. The extracted protein samples were subjected to reductive alkylation treatment to open the disulfide bonds for subsequent enzymatic hydrolysis of the proteins. Trypsin and 8-plex iTRAQ reagent (AB Sciex, Cat. No. 4381664) were used to label the protein. The mixed peptides were preisolated using high $\mathrm{pH}$ reverse phase chromatography, and liquid chromatography was performed coupled with tandem mass spectrometry (LC-MS/MS) analysis. The mass spectrometry data was assessed using Protein Pilot software (AB, Version 5.0) and aligned for identification; the database used was UniProtKB/Swiss-Prot. The identification criteria for the differentially expressed proteins had a fold difference of $\geq 1.5$ or $\leq 0.667$, and the number of unique peptides per protein $\geq 2$ and $<0.05$ was considered to be a significant difference.
The OmicsBean (https://www.omicsbean.cn/) multifunctional bioinformatics analysis tool, integrated STRING (https://www.string-db.org) biological database, and Cytoscape software were used to perform enrichment analysis on the identified differentially expressed proteins based on GO biological process, cellular component, and molecular function. Additionally, KEGG (https:/www.kegg.jp/kegg/ pathway.html) biological pathway enrichment analysis was performed on the differentially expressed proteins.

\subsection{HPLC Fingerprinting and Active Ingredient Detection.} A reference solution that contained notoginsenoside R1, ginsenoside Rg1, ginsenoside Re, icariin, and ginsenoside $\mathrm{Rb} 1$ was mixed with methanol at a concentration of $0.1 \mathrm{mg} / \mathrm{mL}$. The ZJP powder was dissolved in $100 \%$ methanol and filtered as test solution. The reference solution and the test solution were tested via HPLC, and the characteristic maps of both solutions were obtained. The chromatographic conditions were as follows: octadecyl silane-bonded silica gel was used as filler, and gradient elution was carried out at a detection wavelength of $203 \mathrm{~nm}$. The column was an Agilent TC-C18 $(250 \mathrm{~mm} \times 4.6 \mathrm{~mm}, 5 \mu \mathrm{m})$ with water as mobile phase A and acetonitrile as mobile phase $\mathrm{B}$ (gradient elution: 0-12 $\mathrm{min}, 81 \% \mathrm{~A}$, and $12-60 \mathrm{~min}, 81 \%-64 \% \mathrm{~A}$; flow rate $1.0 \mathrm{~mL} / \mathrm{min}$; and a detection wavelength of $203 \mathrm{~nm}$ ).

2.12. Statistical Analysis. The obtained data were statistically analyzed and processed using State 12.0 software, and the measured data were expressed as the mean \pm standard deviation $(\bar{x} \pm \mathrm{SD})$. One-way analysis of variance (ANOVA) was used to assess the differences between the groups, and the Bonferroni test was used for multiple comparisons between the groups. Wilcoxon rank-sum test was used for comparison between the group data which did not conform to a normal distribution or variance. Statistical significance was defined as $P<0.05$.

\section{Results}

3.1. Body Weight, Fasting Blood Glucose, and Fasting Insulins. The body weight of the model group $(238.7 \pm 22.08 \mathrm{~g})$ was significantly lower than that in the normal group $(408.8 \pm 21.06 \mathrm{~g}) \quad(P<0.01)$. Compared with the model group, the rats in the high-dose ZJP group $(308.3 \pm 19.35 \mathrm{~g})$ and the low-dose ZJP group $(305.9 \pm 13.25 \mathrm{~g})$ had gained significant weight $(P<0.01)$. The results are shown in Figure 1(a). In addition, as shown in Figure 1(b), both the high-dose $(13.16 \pm 2.15 \mathrm{mM})$ and low-dose groups $(14.66 \pm 1.22 \mathrm{mM})$ of ZJP could effectively reduce the fasting blood glucose of the model group $(23.79 \pm 5.54 \mathrm{mM})$ $(P<0.01)$, and there was no significant difference between the two groups. Also, ZJP could significantly improve the fasting insulin of model group $(514.0 \pm 180.7 \mathrm{pg} / \mathrm{mL})$ $(P<0.01)$, while there was no significant difference between high-dose $(2360 \pm 718.2 \mathrm{pg} / \mathrm{mL})$ and low-dose groups $(2508 \pm 925 \mathrm{pg} / \mathrm{mL})$ (Figure 1(c)). 


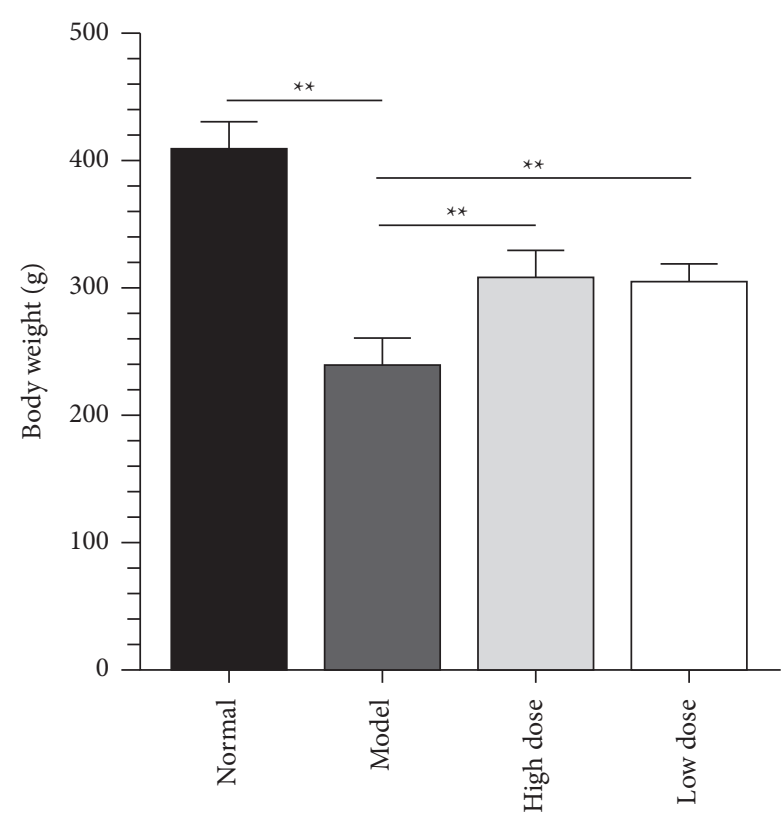

(a)

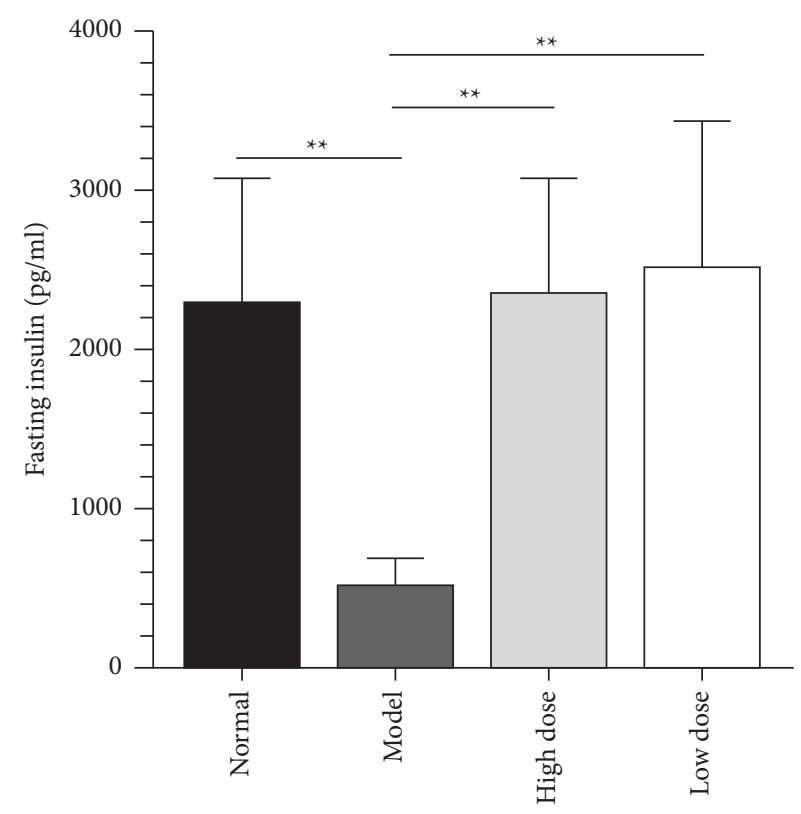

(c)

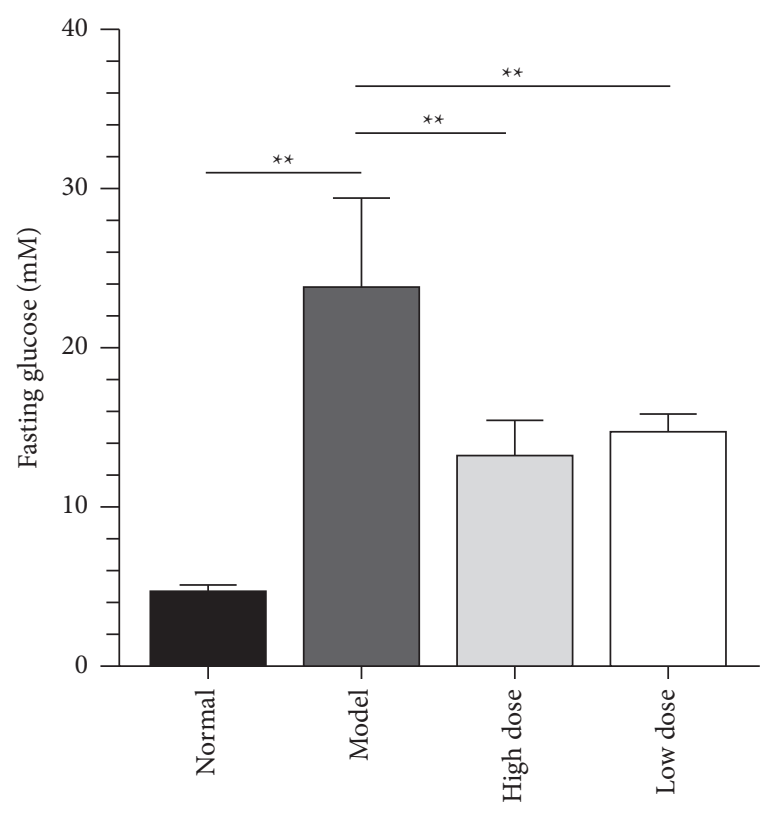

(b)

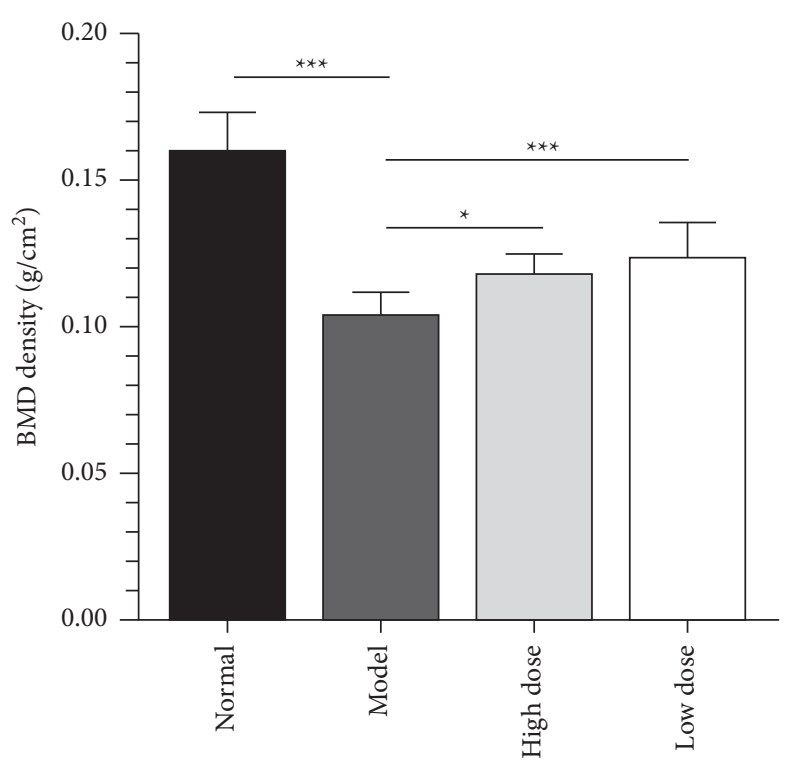

(d)

FIGURE 1: Body weight. (a) Fasting blood glucose and (b) and fasting insulin (c) of each group. (d) BMD.

3.2. ZJP Can Improve the BMD of Lumbar Vertebrae in DOP Model Rats. As shown in Figure 1(d), the BMD of the model group $\left(0.11 \pm 0.01 \mathrm{~g} / \mathrm{cm}^{2}\right)$ was significantly lower than that in the normal group $\left(0.16 \pm 0.01 \mathrm{~g} / \mathrm{cm}^{2}\right)(P<0.001)$. Compared with the model group, the rats in the high-dose ZJP $\left(0.12 \pm 0.01 \mathrm{~g} / \mathrm{cm}^{2}\right)$ and the low-dose ZJP groups $\left(0.13 \pm 0.01 \mathrm{~g} / \mathrm{cm}^{2}\right)$ had proven the BMD of lumbar vertebrae in DOP model rats $(P<0.001$ and $P<0.05$, respectively.).

3.3. ZJP Can Improve the Status of Pancreatic Islet Cells in DOP Model Rats. We assessed the effect of ZJP on pancreatic islet cells by $\mathrm{H} \& \mathrm{E}$ staining, immunofluorescence, and transmission electron microscopy. The islet cells in the normal group were lightly stained, with a high cell density, uniform distribution, and regular morphology (Figures 2(a) and $2(\mathrm{e})$ ). The mitochondria, endoplasmic reticulum, and Golgi were abundant in the cytoplasm of the normal group (Figure 2(i)). In the model group, the islets were significantly reduced, their shape was irregular, their cell density was reduced, and more cells presented with nuclear pyknosis. The peripheral acinar cells also demonstrated substantial atrophy and degeneration (Figures 2(b) and 2(f)). Moreover, the number of mitochondria was decreased, and they were swollen and deformed, the endoplasmic reticulum had expanded to different extents, and a degranulation 

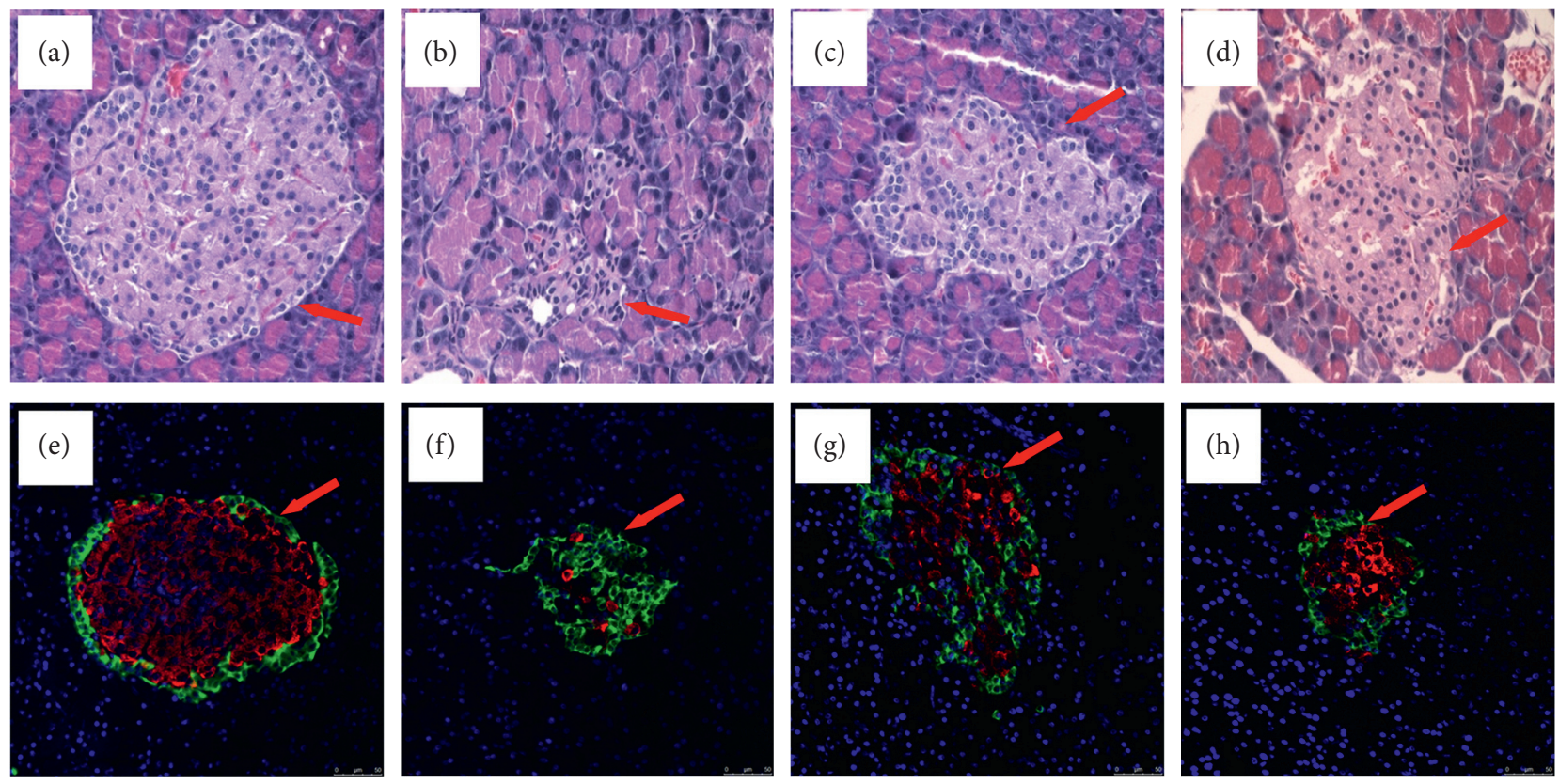

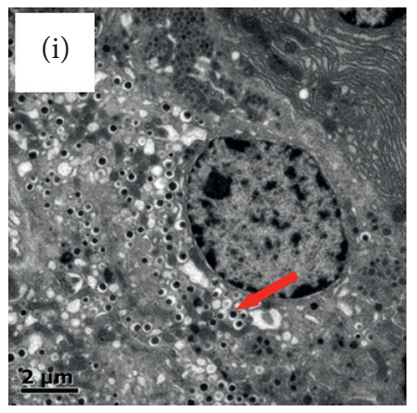

Normal

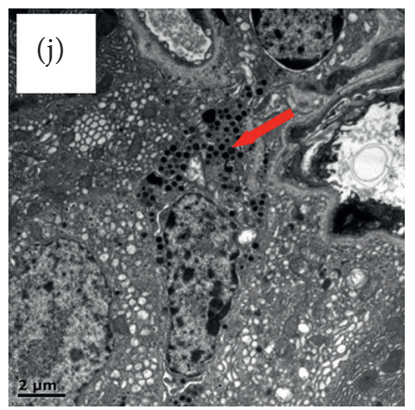

Model

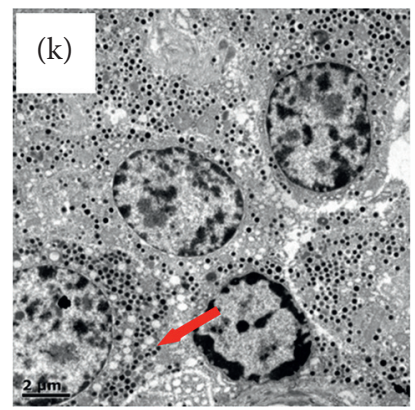

High dose

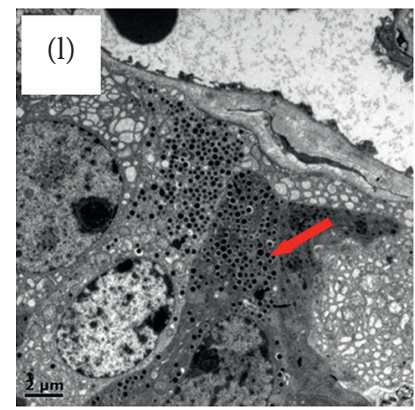

Low dose

Figure 2: Pancreatic microstructure in each group. (a-d) H\&E staining $(\times 400)$; (e-h) insulin-glucagon double-label immunofluorescence $(\times 400)$; (i-l) transmission electron microscopy $(\times 5000)$. A, E, and I: normal group. B, F, and J: model group. C, G, and K: high-dose ZJP group. D, H, and L: low-dose ZJP group.

phenomenon could be seen (Figure 2(j)). The pancreatic islet structures in the high-dose and low-dose ZJP groups were significantly improved compared with the model group. In these treatment groups, the islet size was basically normal, the morphology was regular, the islet cells were arranged neatly, the density was acceptable, and the numbers of atrophied and degenerated islet cells were decreased (Figures 2(c), 2(d), 2(g), and 2(h)). Additionally, in these treatment groups, the endoplasmic reticulum was not significantly expanded (Figures 2(k) and 2(l)).

\subsection{ZJP Can Improve the Histological Morphology of Bone} Tissues in DOP Model Rats. In addition to the effects of bone mass reduction, DOP is also related to bone structure factors and the number of microinjuries in the bone $[20,21]$. Therefore, we performed electron microscopy to assess for these changes in the bone microstructures. The bone density of the femurs in the normal group was thick and the trabecular bones were arranged neatly. The surface of the trabecular bone was regular and smooth, the gap between the trabeculae was small, and the collagen fibers were neatly arranged in the trabecular bone (Figure 3(a)). In the model group, the bone density appeared to be significantly thinner, the marrow cavity was larger, the trabecular bone connection was interrupted, and the reticular structure was destroyed (Figure 3(b)). The trabecular bone structures in the high-dose and low-dose ZJP groups were more organized compared with the model group. The bone marrow cavities were also smaller (Figures 3(c) and 3(d)).

3.5. GO and KEGG Analysis of ZJP Function in DOP Model Rats. In order to better clarify how ZJP exerts its antiosteoporosis function, we carried out proteomic GO and KEGG analyses. We classified the altered proteins after lowdose ZJP treatment into GO classification due to the equal effect with high-dose ZJP treatment on DOP rats. Compared to the normal group, the results in the model group indicated that DOP mostly resulted in protein changes in biological process including anatomical structure development (13\%), response to stress (13\%), biosynthetic process (12\%), and cellular nitrogen compound metabolic process (12\%) (Figure 4(a)). The molecular functions of these proteins most 

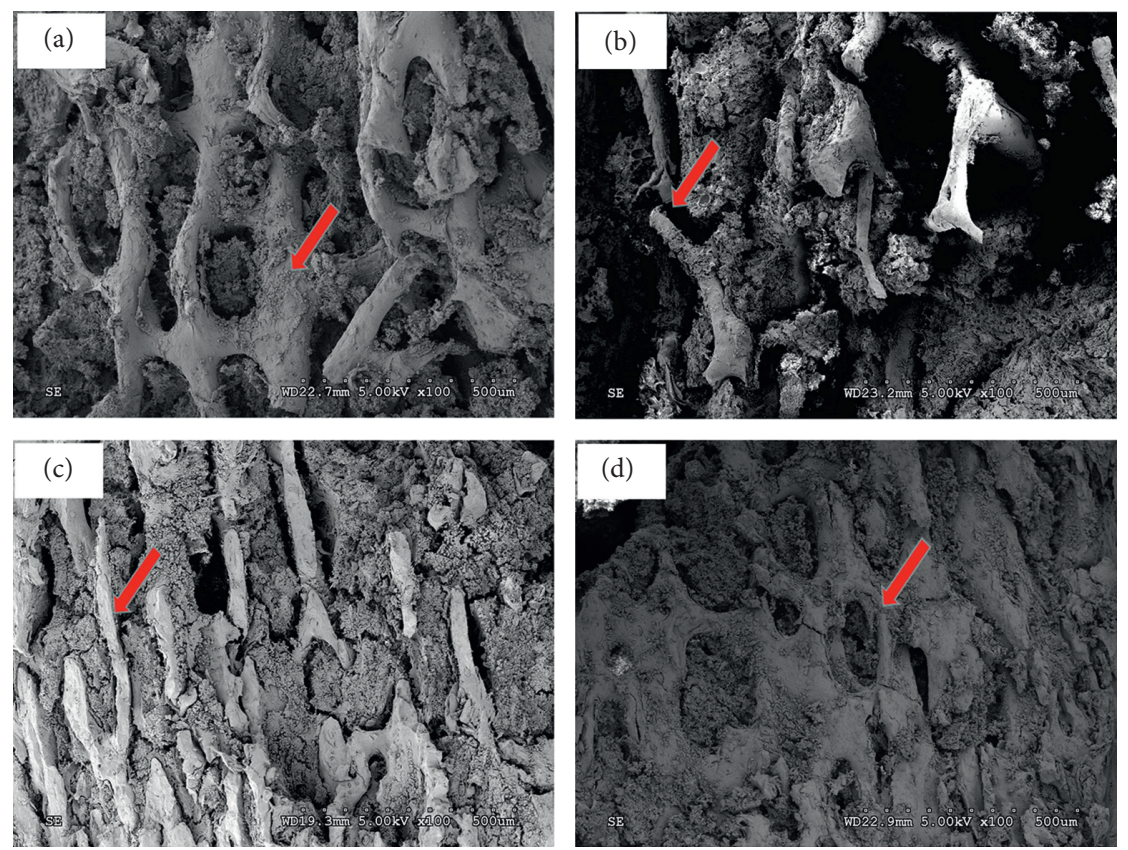

FIGURE 3: Scanning electron micrograph of a femur from each group $(\times 100)$. (a) Normal group. (b) Model group. (c) High-dose ZJP group. (d) Low-dose ZJP group.

likely reflected ion binding (19\%), RNA binding (17\%), and enzyme binding (15\%) (Figure 4(b)). As for the cellular component, the highest probability of existence included the extracellular region (17\%), organelle (17\%), and cytoplasm (16\%) (Figure 4(c)). By comparing the low-dose ZJP group with the model group, we arrived at a similar conclusion with regard to these three categories (Figures 4(d)-4(f)).

We then performed pathway enrichment analysis on the differentially expressed proteins. Comparing the normal group with the model group, the pathways with the highest degree of enrichment involved the following aspects: fatty acid elongation, arginine and proline metabolism, fatty acid degradation, PPAR signaling pathway, fat digestion and absorption, adipocytokine signaling pathway, and DNA replication (Figure 5(a)). Meanwhile, comparing the model group with ZJP treatment groups, the most enriched pathways were ribosome, vitamin digestion and absorption, DNA replication, arginine and proline metabolism, and fat digestion and absorption (Figure 5(b)).

3.6. ZJP Fingerprint and Active Ingredients. We first determined the fingerprint map of ZJP via HPLC and identified its active ingredients (Figure 6). There were five major characteristic peaks in the map with relative retention time specifications, including notoginsenoside R1 (peak 1), ginsenoside Rg1 (peak 2), ginsenoside Re (peak 3), icariin (peak 4), and ginsenoside Rb1 (peak 5).

\section{Discussion}

DOP is a common complication of DM. With the aging of the global population, the incidence of both DM and DOP is becoming higher and is affecting people's quality of life. At present, the treatment for DOP includes three types: supplementation of calcium and vitamin $\mathrm{D}[22,23]$, inhibition of bone resorption with drugs such as bisphosphonates [24-26], and promotion of bone formation with drugs such as PTH1-34 [27, 28]. However, the overall effect of these treatments is not particularly good, with a high rate of side effects and a high cost. Currently, numerous studies are focusing on the effects of TCM with regard to osteoporosis [29-31], but the concise role and mechanism underlying such treatments are still unclear. As such, it is important to understand the molecular mechanism and related pathogenesis of DOP, so as to help identify an effective therapy. This study found that ZJP can significantly improve the BMD of lumbar vertebrae in DOP model rats, the histomorphology, and ultrastructure of bone and pancreatic islet tissues. After further proteomic analysis of differentially expressed proteins, it is found that ZJP can exert its effects by affecting various aspects of glucose metabolism and bone metabolism. The influence of DOP, which involves multiple pathways, indicates that ZJP, a traditional Chinese medicine compound, plays a role through the characteristics of multiple components and multiple targets and may be used as an effective alternative drug for the treatment of DOP.

A previous study has demonstrated that ZJP can effectively improve glucose metabolism and abnormal bone metabolism and regulate blood and urinary metabolism in DOP rats [15]. ZJP has a clear hypoglycemic and lipidlowering effect and can improve insulin sensitivity, promote resistance to oxidation, protect the vascular endothelium, and reduce the level of inflammatory factors $[32,33]$. Based on the establishment of our DOP rat model, we found that ZJP could reduce the weight loss of rat caused by DOP and improve the fasting blood glucose and fasting insulin of these rats (Figure 1). The most important changes occurring 


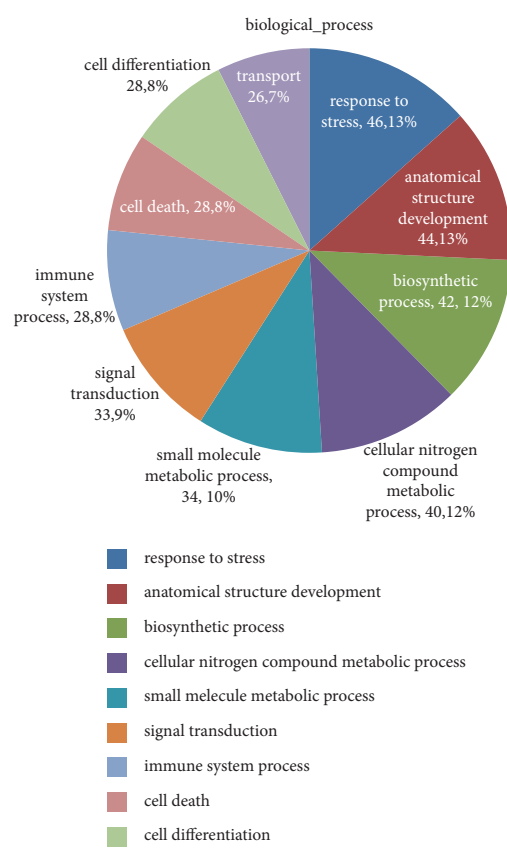

(a)

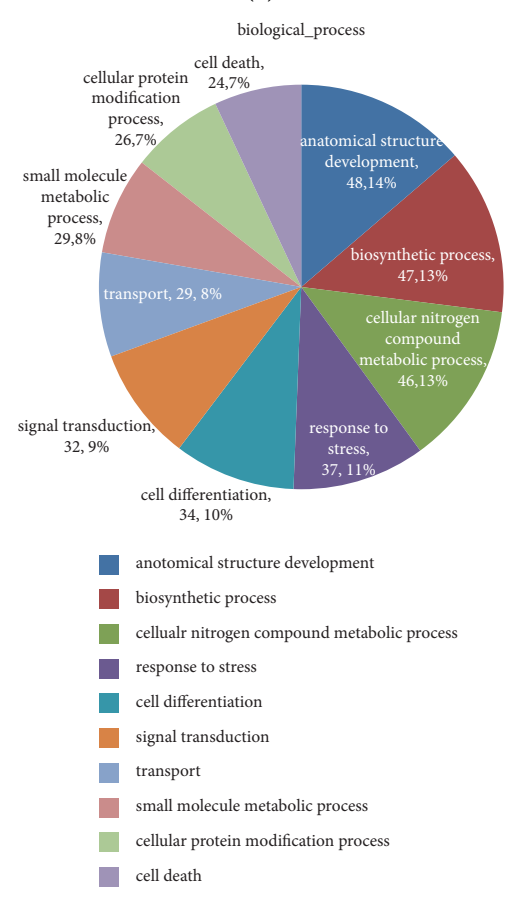

(d)

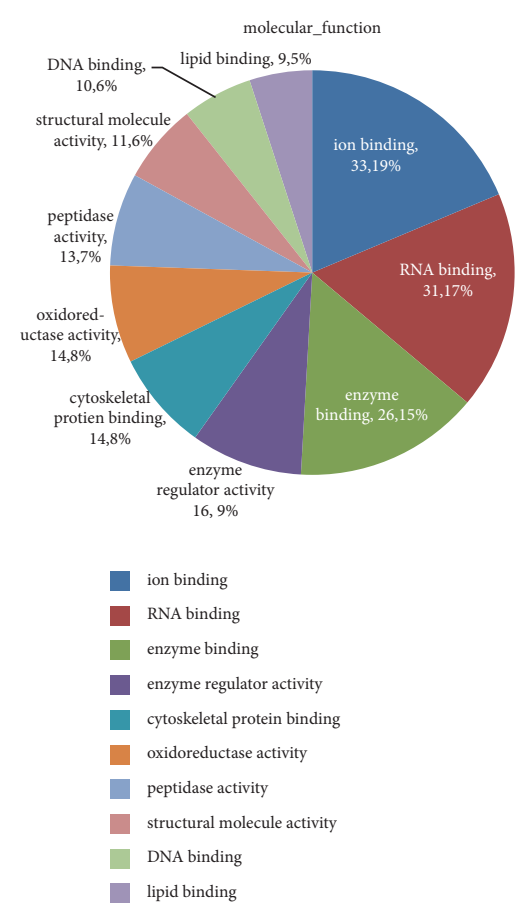

(b)

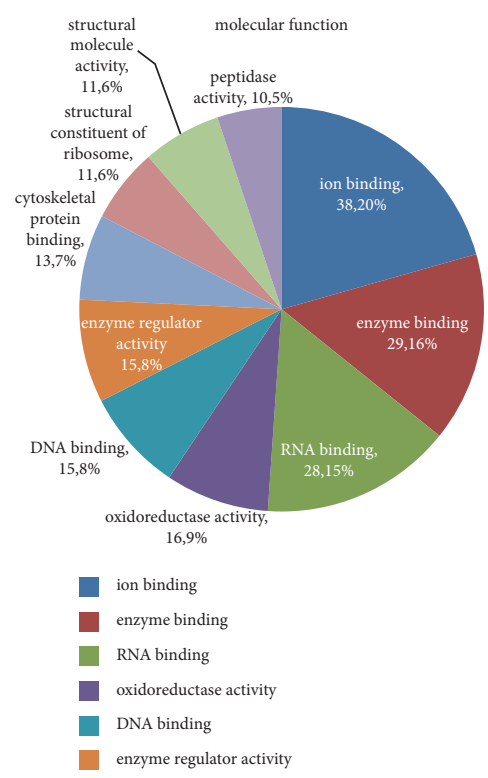

(e)

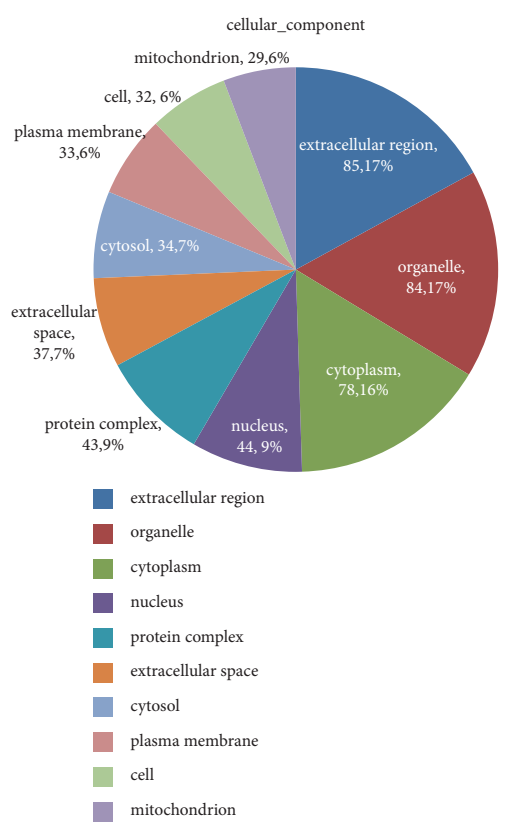

(c)

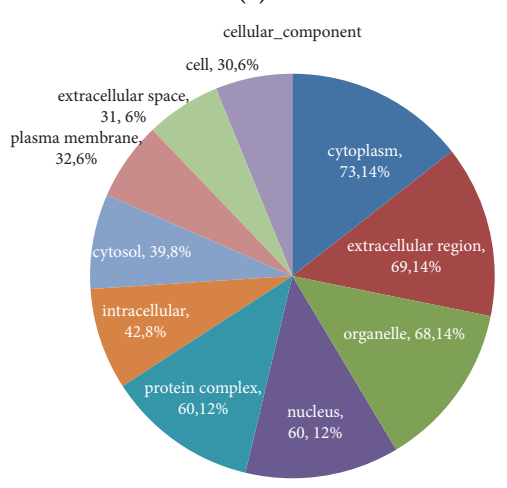

cytoplasm

- extracellular region

organelle

nucleus

protein complex

intracellular

cytosol

plasma membrane

extracellular space

cell

FIGURE 4: GO analysis results for the different groups of DOP rats. The normal group versus model group: (a) biological process, mainly differenced in response to stress, anatomical structure development, and biosynthetic process; (b) molecular function, mainly differenced in ion binding, RNA binding, and enzyme binding; (c) cellular component, mainly differenced in the extracellular region, organelle, and cytoplasm. The model group versus low-dose ZJP group: (d) biological process, mainly differenced in anatomical structure development, biosynthetic process, and cellular nitrogen compound metabolic process; (e) molecular function, mainly differenced in ion binding, enzyme binding, and RNA binding; (f) cellular component, mainly differenced in the cytoplasm, extracellular region, and organelle.

in patients with DOP involve alterations in blood glucose and the skeletal system. Therefore, microscopic analyses of islet cells and bone tissues were performed in the present study. The results indicated that ZJP conferred an obvious improvement to the histomorphology and ultrastructure of islets (Figure 2) and could improve bone formation, reduce bone resorption, increase bone density, and improve the bone microstructure (Figure 3). These pathology results 


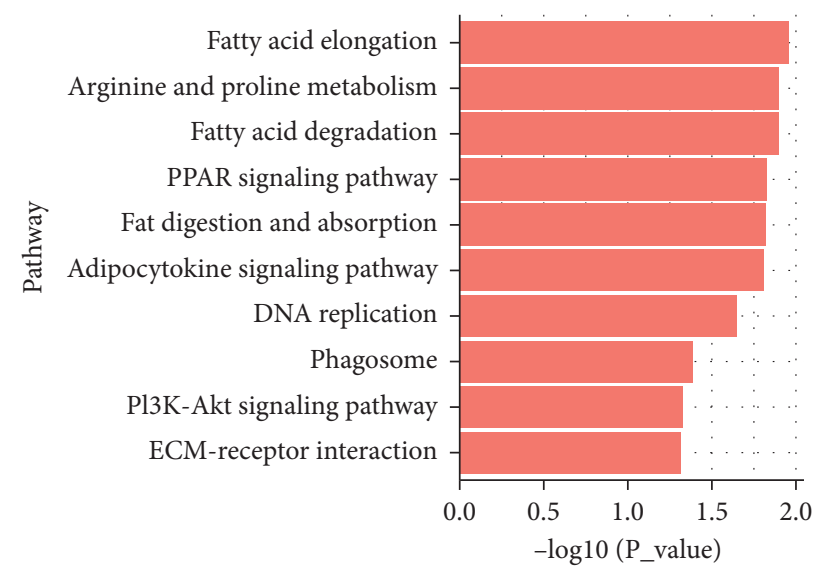

(a)

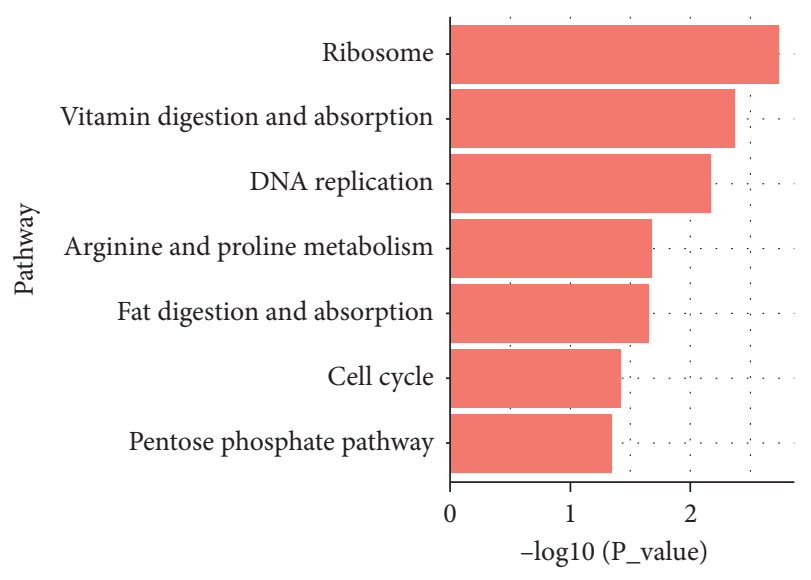

(b)

Figure 5: Pathway analysis of the differentially expressed proteins between the different groups. (a) Normal group versus model group, mainly in fatty acid elongation, arginine and proline metabolism, and fatty acid degradation pathway. (b) Model group versus low-dose ZJP group, mainly in ribosome, vitamin digestion and absorption, and DNA replication pathway.

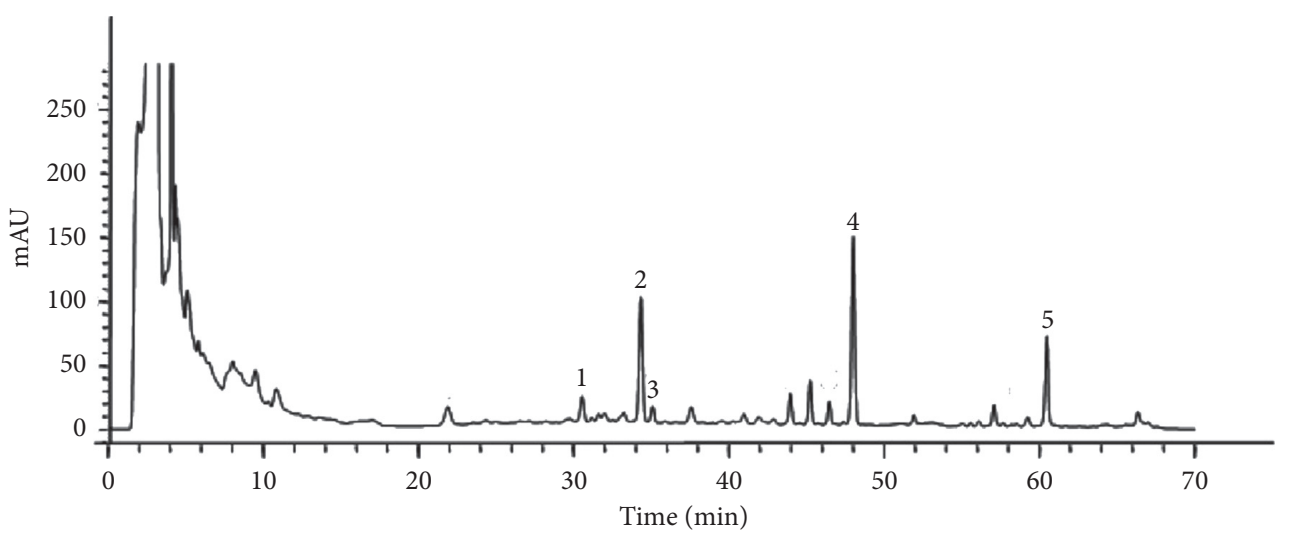

Peak 1: notoginsenoside R1

Peak 2: ginsenoside Rg1

Peak 3: ginsenoside Re

Peak 4: icariin

Peak 5: ginsenoside Rb1

Figure 6: HPLC fingerprint of ZJP. Peak 1: notoginsenoside R1; peak 2: ginsenoside Rg1; peak 3: ginsenoside Re; peak 4: icariin; peak 5: ginsenoside Rb1.

support our previous findings [15] that ZJP was shown to exert its influence by affecting aspects of glucose metabolism and bone metabolism.

To further investigate the potential mechanism of ZJP in the treatment of DOP, we performed proteomic GO and KEGG analyses on the identified differentially expressed proteins. The results revealed that ZJP most likely affected ion binding, RNA binding, and enzyme binding involved in several biological processes, including anatomical structure development, response to stress, biosynthetic processes, and cellular nitrogen compound metabolic processes. The roles of ZJP in these aspects are closely related to known factors related to the occurrence and development of DOP. Moreover, these roles are reflected in several aspects of DOP, such as bone structure changes and blood metabolism. The highest probabilities of existence with regard to the cellular component were the extracellular region, organelles, and cytoplasm, indicating that it is possible that ZJP might have some functions affecting the cellular microenvironment. The enrichment pathways identified mostly referred to the ribosome, vitamin digestion and absorption, DNA replication, arginine and proline metabolism, and fat digestion and absorption, indicating that ZJP is closely related to changes in body weight and calcium absorption as well as protein synthesis. However, to further characterize the protein signaling network associated with the treatment of DOP via ZJP, it will be necessary to further identify the specific target genes via additional methods, both in vitro and in vivo, in an effort to define the mechanism of action of ZJP with regard to the treatment of DOP. 
ZJP is a Chinese herbal compound developed by Shenzhen TCM Hospital, and this study was the first to define the fingerprint map of ZJP via HPLC and ascertain the primary active ingredients, including notoginsenoside R1, ginsenosideRg1, ginsenoside $\mathrm{Re}$, icariin, and ginsenoside $\mathrm{Rb} 1$. Thus, notoginsenoside R1, ginsenoside Rg1, ginsenoside $\mathrm{Re}$, icariin, and ginsenoside Rb1 together were found to have both an antihypoglycemic action and an antiosteoporosis effect in the previous study [34, 35]. Pharmacological studies have confirmed that epimedium extracts can inhibit the activity of osteoclasts via various means and promote the differentiation of osteoblasts and increased bone formation and bone density [36-38]. Ginsenoside Rg1 treatment of diabetic rats was associated with reduced oxidative stress and attenuated myocardial apoptosis [35]. Icariin could significantly regulate the nNOS and calponin in penile tissues of all rats [34]. Icariin attenuates titaniumparticle inhibition of bone formation by activating the Wnt/ $\beta$-catenin signaling pathway in vivo and in vitro [37]. These observations provided theoretical support for the prevention and treatment of DOP by ZJP.

\section{Conclusions}

Overall, this study identified the main active components of ZJP and determined that ZJP could significantly improve the histomorphology and ultrastructure of bone and islets tissues using a DOP rat model and might serve as an effective alternative medicine for the treatment of DOP. In the further study, we will focus on one compound as a representative ingredient and verify the predicted mechanisms.

\section{Data Availability}

All the data and materials of this manuscript are available.

\section{Ethical Approval}

This manuscript was in accordance with the Animal Research Ethics Committee and approved by the Animal Protection and Use Committee of the Guangdong Experimental Animal Center (approval number: IACUC-G16003).

\section{Disclosure}

This manuscript has been submitted as a preprint in the following link: https://www.researchsquare.com/article/rs$63628 / \mathrm{v} 1$.

\section{Conflicts of Interest}

The authors declare that they have no conflicts of interest related to this work.

\section{Authors' Contributions}

Chu SF and Li HL designed the study. Chu SF, Liu DL, Zhao HX, Shao MM, Chen JP, Liu XM, and Qu X performed the research. Liu DL, Li ZY, and Li JH analyzed data. Chu SF and
Li HL wrote the paper. The manuscript was approved by all authors for publication.

\section{Acknowledgments}

The study was supported by the Shenzhen Municipal Science and Technology Bureau (JCYJ20140408153331810 and JCYJ20170817094838619) and Sanming Project of Medicine in Shenzhen (SZSM201512043).

\section{References}

[1] M. Lechleitner, K. Pils, R. Roller-Wirnsberger et al., "Diabetes and osteoporosis: pathophysiological interactions and clinical importance for geriatric patients," Zeitschrift für Gerontologie und Geriatrie, vol. 46, no. 5, pp. 390-397, 2013.

[2] C. Hamann, S. Kirschner, K.-P. Günther, and L. C. Hofbauer, "Bone, sweet bone--osteoporotic fractures in diabetes mellitus," Nature Reviews Endocrinology, vol. 8, no. 5, pp. 297-305, 2012.

[3] A. Montagnani, S. Gonnelli, M. Alessandri, and R. Nuti, "Osteoporosis and risk of fracture in patients with diabetes: an update," Aging Clinical and Experimental Research, vol. 23, no. 2, pp. 84-90, 2011.

[4] S. Kurra and E. Siris, "Diabetes and bone health: the relationship between diabetes and osteoporosis-associated fractures," Diabetes/Metabolism Research and Reviews, vol. 27, no. 5, pp. 430-435, 2011.

[5] A. Montagnani and S. Gonnelli, "Antidiabetic therapy effects on bone metabolism and fracture risk," Diabetes, Obesity and Metabolism, vol. 15, no. 9, pp. 784-791, 2013.

[6] P. Vestergaard, "Discrepancies in bone mineral density and fracture risk in patients with type 1 and type 2 diabetes--a meta-analysis," Osteoporosis International, vol. 18, no. 4, pp. 427-444, 2007.

[7] A. V. Schwartz, "Efficacy of osteoporosis therapies in diabetic patients," Calcified Tissue International, vol. 100, no. 2, pp. 165-173, 2017.

[8] T. Wang, L. Cai, Y. Wang et al., "The protective effects of silibinin in the treatment of streptozotocin-induced diabetic osteoporosis in rats," Biomedicine \& Pharmacotherapy, vol. 89, pp. 681-688, 2017.

[9] B. Chang, Q. Quan, Y. Li, H. Qiu, J. Peng, and Y. Gu, "Treatment of osteoporosis, with a focus on 2 monoclonal antibodies," Medical Science Monitor, vol. 24, pp. 8758-8766, 2018.

[10] J. C. Gallagher, "Advances in osteoporosis from 1970 to 2018," Menopause, vol. 25, no. 12, pp. 1403-1417, 2018.

[11] T. Suzuki, Y. Nakamura, and H. Kato, "Vitamin D and calcium addition during denosumab therapy over a period of four years significantly improves lumbar bone mineral density in Japanese osteoporosis patients," Nutrients, vol. 10, 2018.

[12] C. Choi, H. Lee, H. Lim, S. Park, J. Lee, and S. Do, "Effect of Rubus coreanus extracts on diabetic osteoporosis by simultaneous regulation of osteoblasts and osteoclasts," Menopause, vol. 19, no. 9, pp. 1043-1051, 2012.

[13] W. Qi, Y. Zhang, Y. B. Yan et al., "The protective effect of cordymin, a peptide purified from the medicinal mushroom cordyceps sinensis, on diabetic osteopenia in alloxan-induced diabetic rats," Evidence-based Complementary and Alternative Medicine, vol. 2013, Article ID 985636, 2013.

[14] X.-J. Li, Z. Zhu, S.-L. Han, and Z.-L. Zhang, "Bergapten exerts inhibitory effects on diabetes-related osteoporosis via the regulation of the PI3K/AKT, JNK/MAPK and NF-kappaB 
signaling pathways in osteoprotegerin knockout mice," International Journal of Molecular Medicine, vol. 38, no. 6, pp. 1661-1672, 2016.

[15] H. Li, S. Chu, H. Zhao et al., "Effect of zishen Jiangtang Pill, a Chinese herbal product, on rats with diabetic osteoporosis," Evidence-based complementary and alternative medicine, vol. 2018, Article ID 7201914, 2018.

[16] J. Chen, L. Zheng, Z. Hu et al., "Metabolomics reveals effect of zishen Jiangtang Pill, a Chinese herbal product on high-fat diet-induced type 2 diabetes mellitus in mice," Frontiers in Pharmacology, vol. 10, p. 256, 2019.

[17] G. Kizilay, O. Ersoy, A. Cerkezkayabekir, and Y. TopcuTarladacalisir, "Sitagliptin and fucoidan prevent apoptosis and reducing ER stress in diabetic rat testes," Andrologia, vol. 53, no. 3, Article ID e13858, 2021.

[18] S. Qi, J. He, H. Han et al., "Anthocyanin-rich extract from black rice (Oryza sativa L. Japonica) ameliorates diabetic osteoporosis in rats," Food \& Function, vol. 10, no. 9, pp. 5350-5360, 2019.

[19] X. Ying, X. Chen, T. Wang, W. Zheng, L. Chen, and Y. Xu, "Possible osteoprotective effects of myricetin in STZ induced diabetic osteoporosis in rats," European Journal of Pharmacology, vol. 866, Article ID 172805, 2020.

[20] Y. H. An, J. Zhang, Q. Kang, and R. J. Friedman, "Mechanical properties of rat epiphyseal cancellous bones studied by indentation testing," Journal of Materials Science: Materials in Medicine, vol. 8, no. 8, pp. 493-495, 1997.

[21] L. M. Coe, R. Irwin, D. Lippner, and L. R. McCabe, "The bone marrow microenvironment contributes to type I diabetes induced osteoblast death," Journal of Cellular Physiology, vol. 226, no. 2, pp. 477-483, 2011.

[22] A. C. Ross, J. E. Manson, S. A. Abrams et al., "The 2011 report on dietary reference intakes for calcium and vitamin D from the Institute of Medicine: what clinicians need to know," Journal of Clinical Endocrinology \& Metabolism, vol. 96, no. 1, pp. 53-58, 2011.

[23] K. Li, R. Kaaks, J. Linseisen, and S. Rohrmann, “Associations of dietary calcium intake and calcium supplementation with myocardial infarction and stroke risk and overall cardiovascular mortality in the Heidelberg cohort of the European Prospective Investigation into Cancer and Nutrition study (EPIC-Heidelberg)," Heart, vol. 98, no. 12, pp. 920-925, 2012.

[24] L. M. Coe, S. A. Tekalur, Y. Shu, M. J. Baumann, and L. R. McCabe, "Bisphosphonate treatment of type I diabetic mice prevents early bone loss but accentuates suppression of bone formation," Journal of Cellular Physiology, vol. 230, no. 8, pp. 1944-1953, 2015.

[25] Y. Takeuchi, "Innovation of bisphosphonates for improvement of adherence," Clinical Calcium, vol. 27, pp. 197-202, 2017.

[26] S. Davis, M. Martyn-St James, J. Sanderson et al., "A systematic review and economic evaluation of bisphosphonates for the prevention of fragility fractures," Health Technology Assessment, vol. 20, no. 78, pp. 1-406, 2016.

[27] K. Suzuki, N. Miyakoshi, T. Tsuchida, Y. Kasukawa, K. Sato, and E. Itoi, "Effects of combined treatment of insulin and human parathyroid hormone(1-34) on cancellous bone mass and structure in streptozotocin-induced diabetic rats," Bone, vol. 33, no. 1, pp. 108-114, 2003.

[28] T. Tsuchida, K. Sato, N. Miyakoshi et al., "Histomorphometric evaluation of the recovering effect of human parathyroid hormone (1-34) on bone structure and turnover in streptozotocin-induced diabetic rats," Calcified Tissue International, vol. 66, no. 3, pp. 229-233, 2000.
[29] D. Ju, M. Liu, H. Zhao, and J. Wang, "Mechanisms of "kidney governing bones" theory in traditional Chinese medicine," Frontiers of Medicine, vol. 8, no. 3, pp. 389-393, 2014.

[30] Y. Li, S.-S. Lü, G.-Y. Tang et al., "Effect of Morinda officinalis capsule on osteoporosis in ovariectomized rats," Chinese Journal of Natural Medicines, vol. 12, no. 3, pp. 204-212, 2014.

[31] N. Lai, Z. Zhang, B. Wang et al., "Regulatory effect of traditional Chinese medicinal formula Zuo-Gui-Wan on the Th17/Treg paradigm in mice with bone loss induced by estrogen deficiency," Journal of Ethnopharmacology, vol. 166, pp. 228-239, 2015.

[32] X. Yongping, G. Bin, and L. Yingrong, "Effect of zishen Jiangtang Pill on insulin sensitivity in high-fat mouse model and its mechanism," Chinese Medicine Guide, vol. 10, pp. 7-9, 2012.

[33] G. Yunshan, L. Huilin, and Z. Hengxia, "Effects of zishen Jiangtang Pill on serum adiponectin and vascular endothelial function in patients with diabetic macroangiopathy," China Medical Herald, vol. 5, pp. 18-20, 2008.

[34] A. W. Shindel, Z.-C. Xin, G. Lin et al., "Erectogenic and neurotrophic effects of icariin, a purified extract of horny goat weed (Epimedium spp.) in vitro and in vivo," The Journal of Sexual Medicine, vol. 7, no. 4, pp. 1518-1528, 2010.

[35] H.-t. Yu, J. Zhen, B. Pang, J.-n. Gu, and S.-s. Wu, "Ginsenoside Rg1 ameliorates oxidative stress and myocardial apoptosis in streptozotocin-induced diabetic rats," Journal of Zhejiang University - Science B, vol. 16, no. 5, pp. 344-354, 2015.

[36] S. Liu, H. Dong, H. Dai, D. Liu, and Z. Wang, "MicroRNA$216 \mathrm{~b}$ regulated proliferation and invasion of non-small cell lung cancer by targeting SOX9," Oncology letters, vol. 15, pp. 10077-10083, 2018.

[37] J. Wang, Y. Tao, Z. Ping et al., "Icariin attenuates titaniumparticle inhibition of bone formation by activating the Wnt/ beta-catenin signaling pathway in vivo and in vitro," Scientific Reports, vol. 6, no. 1, Article ID 23827, 2016.

[38] J. An, H. Yang, Q. Zhang et al., "Natural products for treatment of osteoporosis: the effects and mechanisms on promoting osteoblast-mediated bone formation," Life Sciences, vol. 147, pp. 46-58, 2016. 